\title{
Intervención en comunicación en el trastorno del espectro autista mediante el programa 'More than Words'. Estudio de caso
}

\author{
Inmaculada Baixauli-Fortea, Nerea Gascón-Herranz, Mercedes de Carlos-Isla, Carla Colomer-Diago
}

Introducción. El programa 'More than Words' pretende capacitar a los padres para aprovechar las situaciones cotidianas como entornos de aprendizaje de la comunicación, a través de la instrucción en estrategias de interacción de tipo responsivo.

Objetivo. Describir los efectos de este programa en las habilidades de comunicación de un niño con trastorno del espectro autista y en el lenguaje que sus padres le dirigen.

Pacientes y métodos. Se siguió un diseño de tres fases (preintervención, intervención y postintervención), en el que se midieron las intervenciones responsivas de los padres y los actos comunicativos del niño.

Resultados. La intervención puede modificar el estilo comunicativo de los padres, particularmente cuando la familia recibe las orientaciones de la logopeda. Sin embargo, el carácter responsivo de la comunicación de los padres tiende a disminuir cuando finaliza la intervención. Por su parte, los actos comunicativos del niño se incrementan, con un tamaño medio del efecto del tratamiento.

Conclusiones. El programa 'More than Words' puede ser un buen punto de partida para que los padres conozcan estrategias que promuevan la comunicación con sus hijos con trastorno del espectro autista.

Palabras clave. Comunicación. Estilo responsivo. Intervención temprana. Trastorno del espectro autista.

\section{Introducción}

Los trastornos del espectro autista (TEA) constituyen alteraciones del neurodesarrollo caracterizadas por dificultades en la comunicación social y la conducta [1]. En la actualidad se dispone de una amplia evidencia empírica que respalda la efectividad de la intervención precoz en niños con TEA o indicadores de riesgo. Los estudios de revisión sistemática y metaanálisis que han analizado los efectos de las intervenciones mediadas por padres han constatado resultados prometedores, aunque poco concluyentes. Oono et al [2] hallaron evidencias de efectividad en indicadores relativos a la interacción padre/ madre-niño, así como en comprensión del lenguaje y reducción de la gravedad del autismo. También el estudio de Beaudoin et al [3] concluyó que los padres eran capaces de aprender e implementar estrategias para estimular el desarrollo de sus hijos con TEA. Sin embargo, los avances en las habilidades de los niños fueron escasos. El reciente metaanálisis de Nevill et al [4] identificó progresos, aunque limitados, en la gravedad de la sintomatología autista, la socialización, la cognición y la comunicación. Precisamente, el área comunicativa constituye un objetivo de intervención esencial, dado que es un déficit nuclear en la propia conceptualización del trastorno y una de las variables relacionadas con evoluciones óptimas a largo plazo.

Las intervenciones en comunicación mediadas por padres se fundamentan en las teorías evolutivas sociointeractivas y transaccionales, que plantean la adquisición del lenguaje como un proceso que ocurre a través de interacciones recíprocas entre padres y niños. Estos enfoques se dirigen a la modificación de una parte del sistema (las conductas verbales de los padres) para facilitar el cambio en la otra parte del sistema (el lenguaje del niño). En este proceso de cambio de estilo comunicativo cobra especial relevancia la noción de estilo responsivo verbal, referido de forma amplia a los comentarios que siguen la iniciativa y la atención del niño y son contingentes a sus actos comunicativos [5]. Estudios correlacionales han revelado que los enunciados verbales responsivos facilitan el aprendizaje inicial del lenguaje en niños con TEA, incluso en aquellos mínimamente verbales [6].

\section{Programa "More than Words"}

El programa 'More than Words' (MTW) [7], dise-
Departamento de Ciencias de la Ocupación, Logopedia, Psicología Evolutiva y de la Educación; Universidad Católica de Valencia; Valencia (I. Baixauli-Fortea, N. Gascón-Herranz). BLOEM, Formación para Familias de Niños con Autismo; Valencia (M. de CarlosIsla). Departamento de Educación; Universitat Jaume I; Castellón, España (C. Colomer-Diago).

Correspondencia:

Dra. Inmaculada Baixauli Fortea. Departamento de Ciencias de la Ocupación, Logopedia, Psicología Evolutiva y de la Educación. Unidad de Autismo. Universidad Católica de Valencia. Campus Capacitas. Guillem de Castro, 175. E-46008 Valencia.

E-mail:

inmaculada.baixauli@ucv.es

Declaración de intereses:

Las autoras declaran la inexistencia de conflictos de interés en relación con este artículo.

Aceptado tras revisión externa: 10.01.18.

Cómo citar este artículo: Baixauli-Fortea I, Gascón-Herranz N, De Carlos-Isla M, Colomer-Diago C. Intervención en comunicación en el trastorno del espectro autista mediante el programa 'More than Words'. Estudio de caso. Rev Neurol 2018; 66 (Supl 1): S77-82.

(c) 2018 Revista de Neurología 
ñado en el centro Hanen de Toronto (Canadá), adopta esta perspectiva. Uno de sus objetivos es capacitar a los padres para aprovechar las situaciones cotidianas como entornos privilegiados para la adquisición del lenguaje. Durante el programa, los padres conocen el estilo de aprendizaje de su hijo y sus preferencias sensoriales, comprendiendo mejor sus fortalezas y dificultades. Además, aprenden el significado de la atención conjunta y su impacto en el desarrollo de la comunicación, el rol del juego, la necesidad de manipular el entorno para motivar al niño a comunicarse, la importancia de la participación activa del niño en episodios frecuentes y extensos de toma de turnos, etc. También aprenden a aplicar estrategias de interacción responsiva ligadas a los objetivos comunicativos del niño.

Durante el programa, que consta de ocho sesiones grupales de aproximadamente dos horas y media de duración, se utiliza el manual More than Words [7]. Las sesiones incluyen presentaciones interactivas, discusiones en grupo, análisis de grabaciones y oportunidades para la práctica. Además, se llevan a cabo tres visitas individuales en las que se graba la interacción entre los padres y el niño. En ellas se controla la aplicación de estrategias, se proporciona feedback a la familia y se constata el progreso experimentado.

Hasta la fecha, se han realizado cuatro estudios sobre la eficacia del programa MTW. El primer trabajo cuasiexperimental fue llevado a cabo por McConachie et al [8], quienes hallaron un incremento significativo en el vocabulario de los niños y una mejora importante en el estilo responsivo de los padres. En este estudio no se emplearon técnicas microanalíticas de las interacciones comunicativas, limitación que trató de subsanar la investigación posterior de Girolametto et al [9], quienes estudiaron los efectos del programa en tres niños pequeños con autismo y sus familias y hallaron un aumento de actos comunicativos responsivos en los padres y del léxico e iniciativa social en los niños.

El primer estudio controlado aleatorizado del programa MTW fue realizado por Carter et al [10]. Los resultados indicaron ganancias en el estilo responsivo parental, cinco y nueve meses después del tratamiento, con notables tamaños del efecto. En cuanto al desarrollo comunicativo de los niños, los progresos estuvieron moderados por sus características pretratamiento (el interés por objetos, por ejemplo). La investigación más reciente fue llevada a cabo por Venker et al [11]. Los padres participantes aumentaron sus conductas verbales responsivas, mientras que los niños avanzaron en actos comunicativos instigados por sus padres.
En nuestro contexto, el programa MTW se viene impartiendo desde hace algunos años y el manual constituye un material de referencia en la intervención temprana en niños con TEA. Sin embargo, no nos consta la existencia de estudios sobre su eficacia en esta población. Dada la influencia de los factores sociales y culturales en el diagnóstico y tratamiento del TEA [12], son necesarias investigaciones que profundicen en la viabilidad y alcance de este tipo de programas en nuestro entorno. El objetivo de este estudio preliminar es precisamente describir las habilidades de comunicación en términos de frecuencia de actos comunicativos en un niño con TEA, antes, durante y después de la implementación de MTW. Además, se analizan los cambios en el lenguaje de los padres dirigido al niño en las interacciones espontáneas grabadas en las tres fases del estudio.

\section{Pacientes y métodos}

\section{Participantes y contexto de la intervención}

El participante fue un niño con diagnóstico de TEA, de 3 años y 5 meses de edad, y sus padres. Se comunicaba de forma no verbal (llevando de la mano, entregando objetos y haciendo un uso instrumental del adulto) y con la emisión de unas cinco aproximaciones a palabras. Para estimar mejor el nivel comunicativo del niño, se administró el Communication and Symbolic Behavior Scales Developmental Profile [13], cuyos resultados fueron los siguientes: escala social (puntuación directa: 10; percentil: 1), escala de habla (puntuación directa: 8; percentil: 16), escala simbólica (puntuación directa: 12; percentil: 5) y escala global (puntuación directa: 30; percentil: 1).

Ambos padres tienen estudios universitarios y un nivel socioeconómico medio.

\section{Diseño}

El estudio siguió un diseño de tres fases: preintervención, intervención y postintervención. Las díadas padres-niño se grabaron en vídeo en la Unidad de Autismo de la Clínica Universitaria de la Universidad Católica de Valencia, lugar donde también se llevó a cabo el programa MTW. Se realizaron cuatro grabaciones, de unos cuatro minutos de duración cada una, en el período preintervención (línea basal), un mes antes del programa, y cuatro grabaciones en la fase de postintervención, un mes después. Estas cuatro grabaciones previas y posteriores a MTW se corresponden con cuatro situa- 
ciones de juego que se propusieron a los padres -juguete favorito, interacción con cuentos, juego físico y rutina diaria (merienda)-. La indicación era que jugaran con su hijo como normalmente lo hacían. Durante el programa, que tiene una duración aproximada de 11 semanas, se realizaron seis grabaciones de cuatro minutos de duración, dos en cada una de las tres visitas individuales, realizadas durante la tercera, la quinta y la séptima semana. En estas grabaciones, los padres proponían actividades y aplicaban las estrategias que se habían abordado en las sesiones grupales, recibiendo feedback de la logopeda, certificada por el centro Hanen para la impartición de MTW. Las grabaciones fueron transcritas y codificadas empleando el programa informático Systematic Analysis of Language Transcripts [14].

\section{Medidas}

Se evaluaron, por un lado, los cambios en el estilo comunicativo de los padres, que fueron los interlocutores en todo el proceso, y por otro, los cambios en el número de actos comunicativos del niño.

Valoración del estilo comunicativo de los padres El lenguaje de los padres fue transcrito y segmentado en intervenciones, definidas como un enunciado o conjunto de enunciados emitidos por un interlocutor y vinculados por una estrategia única de acción e intención [15]. Estas intervenciones se codificaron del siguiente modo con el fin de determinar aquellas con un carácter responsivo [9,11]:

- Comentarios. Son intervenciones que siguen el foco atencional del niño y describen lo que está mirando o con lo que está jugando. También se corresponden con las respuestas verbales a los actos intencionales del niño, en las que se pone en palabras el significado de su acto comunicativo (p. ej.: el niño coge una pieza del rompecabezas de un león y el padre comenta 'león'; el niño sube a una colchoneta y el padre dice 'subir').

- Repeticiones. El interlocutor responde verbalmente a los actos verbales del niño, repitiendo todo o parte de estos enunciados previos (p. ej.: el niño dice 'tes' y el padre repite 'tres').

- Elicitaciones. El interlocutor incita a que el niño se comunique usando claves entonativas o la finalización parcial de oraciones (p. ej., 'uno, dos...). Aunque esta categoría es considerada por algunos autores como directiva [9], se ha incluido en la codificación porque se corresponde con una de las estrategias enseñadas en el programa destinadas a promover la comunicación.
Se computó el porcentaje de emisiones de este tipo sobre el número total de intervenciones en cada sesión, antes, durante y después del tratamiento.

\section{Valoración de la comunicación en el niño}

El número de actos comunicativos expresados por el niño fue codificado de acuerdo con la definición de Wetherby y Prizant [13]. Para que una conducta se considere un acto comunicativo debe ser un gesto, una vocalización o una verbalización, dirigida al adulto con una función comunicativa. Ejemplos de actos comunicativos son elevar los brazos para continuar con un juego o decir 'más' para pedir más saltos.

Las codificaciones del lenguaje de los padres y del niño fueron realizadas por dos de las autoras, que no eran ciegas al propósito del estudio ni al momento temporal en que se llevaron a cabo las grabaciones. Se calculó la fiabilidad entre ambas evaluadoras dividiendo el número de acuerdos por el número de acuerdos más desacuerdos, y multiplicando el número resultante por 100 para obtener una estimación en forma de porcentaje [16]. En el caso de la segmentación en intervenciones de los padres, el acuerdo alcanzó el 95\%. En la identificación de comentarios se obtuvo un 93\% de acuerdo; en las repeticiones, el 100\%, y en las elicitaciones, el 98\%. La codificación de los actos comunicativos del niño alcanzó el 90\%. Los desacuerdos se resolvieron por consenso.

\section{Resultados}

Se calculó el porcentaje de intervenciones responsivas de los padres en las tres fases del estudio, que se registran en la tabla y la figura 1 . El número de actos comunicativos en los tres períodos se representa en la tabla y en la figura 2.

\section{Intervenciones responsivas de los padres}

En la figura 1 se observa un cambio de nivel entre las fases en las intervenciones responsivas de los padres, sobre todo desde la fase de preintervención, en la que la media es de 27,4 (rango: 15,3836,83 ) y la mediana es de 28,69 , a la fase de intervención, en la que la media es de 61,79 (rango: $42,10-92,30)$ y la mediana es de 59,16. El nivel de intervenciones responsivas desciende cuando finaliza el tratamiento, siendo la media de 39,41 (rango: $30,70-51,21$ ) y la mediana de 37,87 . Por tanto, es durante la aplicación del programa cuando se observa un mayor nivel de comunicación responsi- 
Figura 1. Porcentaje de intervenciones responsivas de los padres.

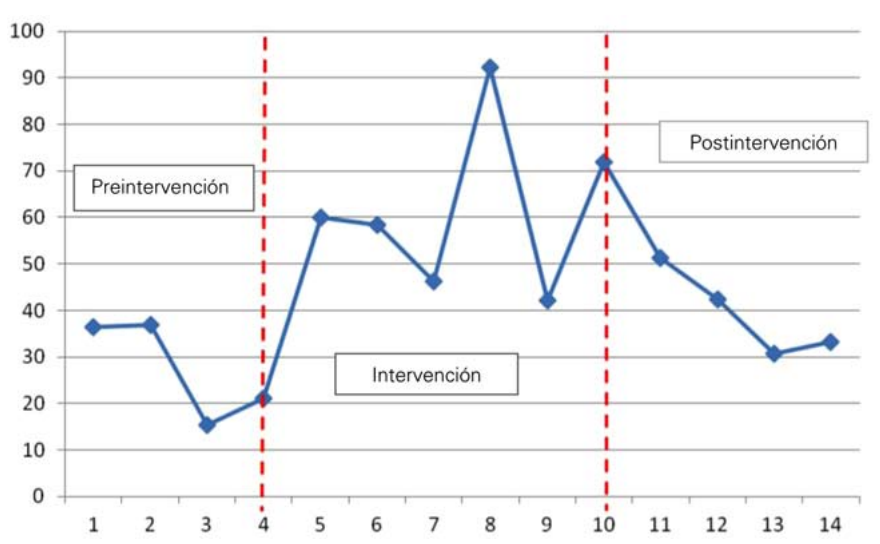

Figura 2. Porcentaje de actos comunicativos del niño.

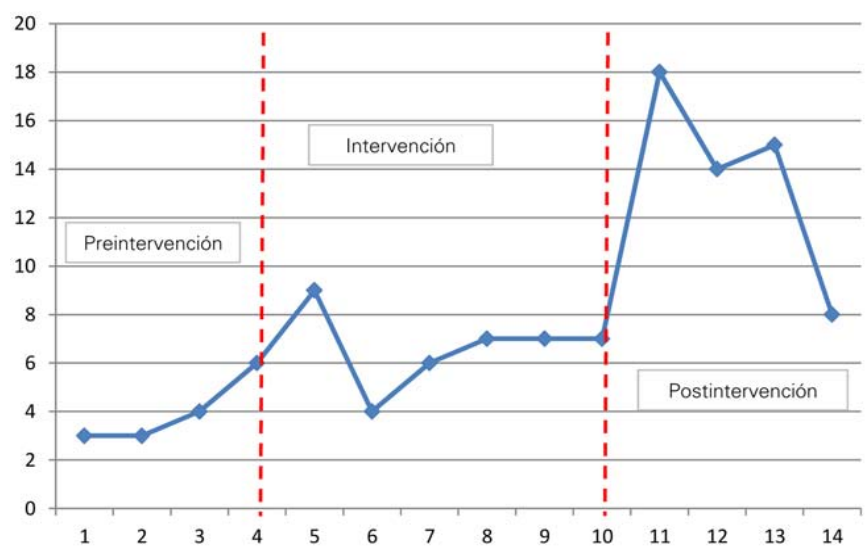

va. En concreto, en la primera grabación del tratamiento se observa un incremento del $38,97 \%$ (de $21,03 \%$ intervenciones responsivas se pasa a un $60 \%$ de intervenciones de este tipo). Es más, a pesar del declive que se aprecia en la cuarta y en la última grabación de la fase de intervención, no llegan a obtenerse valores inferiores a los del período pretratamiento. Sin embargo, en la fase postintervención, una vez se retira el feedback de la terapeuta, el nivel de comunicación responsiva desciende, situándose al final en valores ligeramente inferiores a los más altos de la línea basal. De ello se deduce que el estilo responsivo es máximo cuando los padres reciben asesoramiento por parte de la logopeda, pero tiende a valores que se solapan con los del pretratamiento cuando dejan de recibir este feedback. A pesar de este descenso, los actos comunicativos del niño aumentan de forma notable en esta fase.

\section{Actos comunicativos del niño}

El análisis visual de los datos permite observar un cambio de nivel entre fases, particularmente tras la intervención. Durante el tratamiento, la media es de 6,6 (rango: 4-9) y la mediana es de 7, mientras que en la fase posterior la media de actos comunicativos se sitúa en 13,75 , prácticamente el doble que en la intervención (rango; 7-18) y la mediana, 174,5. El nivel de actos comunicativos aumenta en tres actos con el inicio del tratamiento, si bien se solapa en la tercera y cuarta grabación con valores alcanzados durante la fase preintervención. Es destacable el salto que se produce en los actos comuni- cativos una vez termina el tratamiento, que alcanza valores especialmente elevados en la segunda, tercera y cuarta grabación. Ninguno de los valores de esta fase se equipara o es inferior a los que se observan en línea basal. De ello puede deducirse que tras el tratamiento se mantiene e incluso mejora la comunicación del niño.

Con el objeto de calcular el tamaño del efecto del tratamiento se aplicó el índice de no solapamiento de todos los pares (NAP) [17], dado que no exige un umbral mínimo de mediciones en cada fase. El NAP compara por pares todos los datos de la fase de la línea basal con todos los datos de la fase de tratamiento, por lo que podría interpretarse como el porcentaje de datos sin solapamiento entre las fases de línea basal y tratamiento o el porcentaje de datos que muestran una mejoría respecto a la fase de preintervención. La fórmula que se aplica es la siguiente: $\left(\mathrm{n} .^{\circ}\right.$ de pares sin solapamiento $)+(0,5 \times$ n. ${ }^{\circ}$ de empates) / n. ${ }^{\circ}$ total de pares comparando la línea basal y el tratamiento/seguimiento. En el caso del tamaño del efecto de la intervención, el número total de pares de comparaciones de la preintervención y de la intervención es de $24(4 \times 6)$; en la zona de solapamiento existe un par que muestra descenso (negativo) y dos pares con empate, por lo que encontramos 21 pares sin solapamiento $(24-1-2)$. Aplicando la fórmula, el NAP es igual a 0,92 [21+ $(0,5 \times 2) / 24]$. Tras ajustar el NAP a una escala de $0-100 \%$ [ 1 - $(0,92 / 0,5)]$, el tamaño del efecto es del $84 \%$, lo que se considera un tamaño del efecto medio. En el caso del seguimiento, no existen datos solapados con la preintervención, por lo que el tamaño del efecto es del $100 \%$. 
Tabla. Porcentaje de intervenciones responsivas de los padres y de actos comunicativos del niño.

\begin{tabular}{|c|c|c|c|c|c|c|c|c|c|c|c|c|c|c|}
\hline Padres & 36,36 & 36,83 & 15,38 & 21,03 & 60 & 58,33 & 46,15 & 92,3 & 42,1 & 71,87 & 51,2 & 42,4 & 30,7 & 33,33 \\
\hline Niño & 3 & 3 & 4 & 6 & 9 & 4 & 6 & 7 & 7 & 7 & 18 & 14 & 15 & 8 \\
\hline
\end{tabular}

\section{Discusión}

El objetivo de este estudio preliminar ha sido describir los efectos del programa MTW en las habilidades de comunicación de un niño con TEA y en el lenguaje que sus padres le dirigen. Los resultados indican que la intervención puede modificar el estilo comunicativo de los padres, si bien los datos reflejan una gran heterogeneidad. Esta variabilidad, inherente a la conducta humana, podría atribuirse al tipo de actividades en torno a las cuales interactúan los padres y el niño en cada sesión de la intervención con el fin de aplicar las estrategias abordadas en las sesiones. De hecho, se ha señalado que los padres usan distintos estilos de interacción con diferentes tipos de tareas de juego [18]. La cantidad de intervenciones responsivas es mayor durante el tratamiento, cuando la familia recibe las orientaciones de la logopeda a través del videofeedback. Sin embargo, cuando finaliza la intervención, el estilo responsivo de los padres tiende a disminuir, regresando a niveles similares a los del período preintervención. Ello refleja las dificultades en el mantenimiento de los efectos en el estilo comunicativo de los padres, debido posiblemente a que se trata de conductas verbales automatizadas, difíciles de modificar en el breve tiempo que dura la intervención con MTW. De hecho, algunos estudios refieren que los cambios pueden no observarse de forma inmediata, ya que puede llevar algo de tiempo la interiorización e implementación de las estrategias en diversos contextos cotidianos [10].

Respecto a las habilidades comunicativas del niño, se advierte una mejora en cuatro de las seis grabaciones de la intervención, lo que parece asociarse con un incremento en la comunicación responsiva parental. Sin embargo, resulta llamativo que el mayor ascenso en los actos comunicativos del niño se produzca tras el programa, lo que transcurre de forma paralela con un descenso en las intervenciones responsivas de los padres. Este hecho sugiere que muy probablemente el estilo responsivo no sea el principal factor asociado a cambios en la comunicación. Otras estrategias tratadas en el programa y no analizadas en este estudio (uso de 'tentaciones comunicativas', apoyos visuales, estructuración de las actividades...) pueden haber influido en la cantidad de actos comunicativos expresados por el niño. Es más, no existen criterios definidos sobre el aumento en el estilo responsivo que se requiere para lograr cambios significativos en la comunicación. Aunque numerosas evidencias respaldan una relación entre el estilo responsivo y el desarrollo lingüístico, un estilo directivo puede ser necesario cuando se intenta reconducir la atención de un niño escasamente involucrado o se pretende una mayor participación [19].

En definitiva, y a la vista de los datos obtenidos, la comunicación del niño mejora durante y tras la implementación de MTW. Sin embargo, quedan numerosas cuestiones por dilucidar que puedan explicar las razones para este cambio. Las características del niño y de los padres, las habilidades de la terapeuta o el control de la aplicación de las estrategias instruidas durante el programa son aspectos que merecen una especial consideración.

Hubiera sido deseable un seguimiento más estricto de la fidelidad al tratamiento y la obtención de un mayor número de mediciones de las variables en cada fase. A estas limitaciones se unen amenazas a la validez interna, como la propia maduración del niño o la posible influencia de tratamientos paralelos en otras áreas evolutivas. Además, aunque las muestras espontáneas obtenidas en diferentes contextos proporcionan indicadores excelentes de las habilidades comunicativas del niño, sería aconsejable complementar esta información con los resultados de pruebas estandarizadas y cuestionarios que valoren la comunicación cotidiana y sean, por tanto, más representativos del funcionamiento diario del niño.

Sin duda, otro asunto muy relevante es la validez social del programa en relación con la aceptación y satisfacción de los padres, teniendo en cuenta que MTW se ha desarrollado en un contexto cultural y lingüístico diferente al nuestro. Ambos padres valoraron muy positivamente el programa. Sin embargo, es cierto que la naturaleza indirecta de la intervención comporta un compromiso difícil de asumir por parte de las familias, que tienen ocupaciones labo- 
rales y familiares y necesidades emocionales importantes en el proceso de crianza de un niño con TEA. En este sentido, los padres participantes en el estudio de grupo focal de Patterson y Smith [20] plantearon una serie de propuestas con objeto de mejorar la intervención con MTW. Entre ellas, destacan la pertenencia de los niños al mismo estadio de desarrollo comunicativo, para evitar así sentimientos de frustración; un mayor grado de individualización y de práctica guiada por parte del terapeuta, y la consideración de sesiones de seguimiento una vez finalizado el programa. Por su parte, Carter et al [10] también recomendaron la incorporación de sesiones individuales adicionales para controlar mejor la adhesión al tratamiento y aumentar su intensidad. En cualquier caso, los resultados de este estudio preliminar son prometedores y sugieren que el programa MTW constituye un buen punto de partida para que los padres conozcan estrategias que promuevan la comunicación con sus hijos con TEA.

\section{Bibliografía}

1. Asociación Americana de Psiquiatría. Guía de consulta de los criterios diagnósticos del DSM-5. Arlington, VA: Asociación Americana de Psiquiatría; 2013.

2. Oono IP, Honey EJ, McConachie H. Parent-mediated early intervention for young children with autism spectrum disorders (ASD). Cochrane Database Syst Rev 2013; 30: CD009774.

3. Beaudoin AJ, Sébire G, Couture M. Parent training interventions for toddlers with autism spectrum disorder. Autism Res Treat 2014; 2014: 839890

4. Nevill RE, Lecavalier L, Stratis E. Meta-analysis of parentmediated interventions for young children with autism spectrum disorder. Autism 2016; Nov 14. [Epub ahead of print].

5. McDuffie A, Yoder P. Types of parent verbal responsiveness that predict language in young children with autism spectrum disorder. J Speech Lang Hear Res 2010; 53: 1026-39.
6. Siller M, Sigman M. Modeling longitudinal change in the language abilities of children with autism: parent behaviors and child characteristics as predictors of change. Dev Psychol 2008; 44: 1691-704.

7. Sussman F. More than Words: helping parents promote communication and social skills in children with autism spectrum disorder. Toronto: The Hanen Centre; 1999.

8. McConachie H, Randle V, Hammal D, Le Couteur A. A controlled trial of a training course for parents of children with suspected autism spectrum disorder. J Pediatr 2005; 147: 335-40.

9. Girolametto L, Sussman F, Weitzman E. Using case study methods to investigate the effects of interactive intervention for children with autism spectrum disorders. J Commun Disord 2007; 40: 470-92.

10. Carter A, Messinger D, Stone W, Celimli S, Nahmias A, Yoder P. A randomized controlled trial of Hanen's 'More Than Words' in toddlers with early autism. J Child Psychol Psychiatry 2011; 52: 741-52.

11. Venker CE, McDuffie A, Ellis Weismer S, Abbeduto L. Increasing verbal responsiveness in parents of children with autism: a pilot study. Autism 2012; 16: 568-85.

12. Ennis-Cole D, Durodoye BA, Harris, HL. The impact of culture on autism diagnosis and treatment. Considerations for counselors and other professionals. Fam J 2013; 21: 279-87.

13. Wetherby A, Prizant B. Communication and Symbolic Behavior Scales Developmental Profile (CSBS DP). Baltimore, MD: Brookes Publishing; 2002.

14. Miller J, Chapman R. Systematic analysis of language transcripts. Computer software. Madison: University of Wisconsin; 2000.

15. Briz A. Las unidades de la conversación. In Briz A, Grupo Val.Es.Co, eds. ¿Cómo se comenta un texto coloquial? Madrid: Ariel; 2000. p. 51-79.

16. Sackett C. Observing behavior. Vol. 2. Baltimore, MD: University Park Press; 1978.

17. Parker RI, Vannest K. An improved effect size for single-case research: nonoverlap of all pairs. Behav Ther 2009; 40: 357-67.

18. Deveney S, Cress CJ, Lambert M. Parental directiveness and responsivity toward young children with complex communication needs. Int J Speech Lang Pathol 2016; 18: 53-64.

19. Girolametto L. Reflections on the origins of directiveness: implications for intervention. Journal of Early Intervention 1995; 19: 104-6.

20. Patterson SY, Smith V. The experience of parents of toddlers diagnosed with autism spectrum disorder in the More than Words Parent Education Program. Infants Young Child 2011; 24: 329-43.

\section{A communication intervention in autism spectrum disorder by means of the programme 'More than Words'. A case study}

Introduction. The 'More than Words' programme aims to enable parents to take advantage of day-to-day situations as communication learning settings, through the use of instruction in responsive-type interaction strategies.

Aim. To describe the effects of this programme on the communication skills of a child with autism spectrum disorder and on the language the parents use to address him.

Patients and methods. A three-phase design (pre-intervention, intervention and post-intervention) was employed, in which the responsive interactions of the parents and the child's communicative acts were measured.

Results. The intervention can modify the parents' communicative style, especially when the family receives guidance from a speech therapist. Nevertheless, the responsive nature of the parents' communication tends to diminish when the intervention finishes. Conversely, the child's communicative acts increase, with a medium-sized effect of the treatment.

Conclusions. The 'More than Words' programme can be a good starting point for parents to become familiar with strategies that foster communication with their children who have autism spectrum disorder.

Key words. Autism spectrum disorder. Communication. Early intervention. Responsive style. 